\title{
Procès-verbal \\ de l'assemblée générale d'été de la Société suisse de Chimie
}

\author{
tenue le 8 septembre 1919, à Lugano, \\ sous la présidence de.M. le Professeur Ph.-A. Guye, Président.
}

A. Questions administratives.

1. La Société adhère aux nouveaux statuts de la Société helvétique des Sciences naturelles (S. H. S. N.).

2. M. le Professeur F. Fichter est désigné comme délégué de notre Société au Sénat de la S. H. S. N.

3. Pour tenir compte de l'accroissement du nombre des membres, le comité des Helvetica Chimica Acta a décidé de porter à 1200 exemplaỉres le tirage de cette publication.

4. Les documents qui paraissent déjà dans les Helv. ne seront plus envoyés séparément au membres.

5. La Société est informée de la dissolution de l'Association internationale des Sociétés de Chimie, fondée avant la guerre.

6. Un message de félicitations sera envoyé à M. le Professeur G. Lunge à l'oćcasion de son quatre-vingtième anniversaire.

7. Pour le choix de la localité où sera tenue la prochaine séance d'hiver, la décision est laissée au Comité.

B. Communications scientifiques.

1. Prof. Dr. Fr. Fichter (Basel): Elektrochemische Oxydation des Benzaldehyds.

2. Prof. Dr. Amé Pictet et L. Helfer (Genève): Synthèse dans le groupe de la quinine.

3. Dr. L. Ruzicka (Zürich): Ueber die Selbstkondensation ungesättigter Ketone.

4. Prof. Dr. P. Karrer (Zürich): a) Neues Verfahren zur Herstellung aromatischer Nitrile.

b) Ueber Derıvate der Mandelsäuren.

5. Prof. Dr. E. Briner (Genève): a) Etudes comparatives sur l'électrolyse des chlorures alcalins.

b) Sur la fixation de l'azote atmosphérique sous forme d'acide cyanhydrique(Recherches en collaboration avec M. le Dr. A. Baerfuss).

6. Dr. J. Lifschitz (Zürich): Ueber die refraktometrischen Effekte der Saizbildung.

7. Dr. K. Schweizer" (Bern-Winterthur): L'action du cuivre sur la fermentation alcoolique.

8. Dr. M. Sandoz (Lausanne): Uue réaction paradoxale.

9. Dr. H. Ziegler (Zürich): Ueber die Konstitution der chemischen Atome.

Le Secrétaire: $E$. Briner. 